\title{
Comparison of Three Methods of Predictive Postoperative FEV1 and DLCO Calculations in Relation to Their Observed Postoperative Values in Lung Resection
}

\author{
Vjekoslav Karadža1,*, Ivanka Karadža ${ }^{2}$
}

\author{
${ }^{1}$ Jordanovac Department of Thoracic \\ Surgery, Zagreb University Hospital \\ Center, Zagreb, Croatia \\ ${ }^{2}$ Department of Obstetrics and \\ Gynecology, Varaždin General Hospital, \\ Varaždin, Croatia
}

\section{*Correspondence \\ vkaradza@xnet.hr \\ (Vjekoslav Karadža)}

\begin{abstract}
Introduction: Three ways of simple calculations (segmental based on 18 segments method, segmental based on 19 segments method and subsegmental method) of predictive postoperative values of FEV1 and DLCO are in use during the preoperative survey for patients planned for lung resection as treatment of lung carcinoma as a part of risk assessment. Hypothesis: Segmental calculation method based on 19 segments is better than subsegmental method and segmental calculation method based on 18 segments in prediction of postoperative values of both FEV1 and DLCO one month after lung lobectomy. Materials and methods: Expected postoperative calculated values of FEV1 and DLCO (two segmental and one subsegmental method) of 52 patients undergone lobectomy are related to real postoperative values for same patients one month after surgery. Results: According to univariate analysis, real values of postoperative DLCO correlate most significantly with ppoDLCO calculated by segmental method (18 segments), but real values of postoperative FEV1 correlate most significantly with ppoFEV1 calculated by 19 overall segments segmental method. Data analysis as well showed that preoperative calculated PpoFEV1 and PpoDLCO underestimate real postoperative values of FEV1 and DLCO one month after lobectomy, but it is not statistically significant. Discussion: Same as contemporary guidelines suggest, ppoFEV1 calculation by 19 segments segmental method seems to be the best choice. PpoDLCO is maybe better to calculate by 18 segments segmental method.
\end{abstract}

\section{Keywords}

Thoracic surgery, FEV1, DLCO

\section{Introduction}

Forced expiratory volume in one second (FEV1) and diffusion lung capacity for carbon monoxyde (DLCO) are mainstay of selection for patients undergone lung resection according the literature, including contemporary guidelines $[1-4,6-11]$. It is suggested to calculate expected postoperative values for FEV1 (predicted postoperative FEV1 or PpoFEV1) and DLCO (predicted postoperative DLCO or PpoDLCO) during the preoperative survey for patients planned for lung resection [1-4, 6-11]. The simplest calculations are segmental and subsegmental anatomical meth- ods based on counting of unobstructed pulmonary segments or subsegments to be removed and subtracting that number from number of all nonobstructed pulmonary segments or subsegments of both lungs $[1,3,4,10,11]$. These values are aimed for risk assessment and need for further evaluation [1-4, 6-10]. Patients with low values of PpoFEV1 and/or PpoDLCO are estimated as at high risk for surgery but not necessarily inoperable [1-4, 6-10]. As can be found in literature, right lung is bigger and has upper, middle and lower lobe [12]. Left lung has upper and lower lobe with special segment belonging to upper lobe called lingula with some lobar characteristics [12]. Lobes are divided 


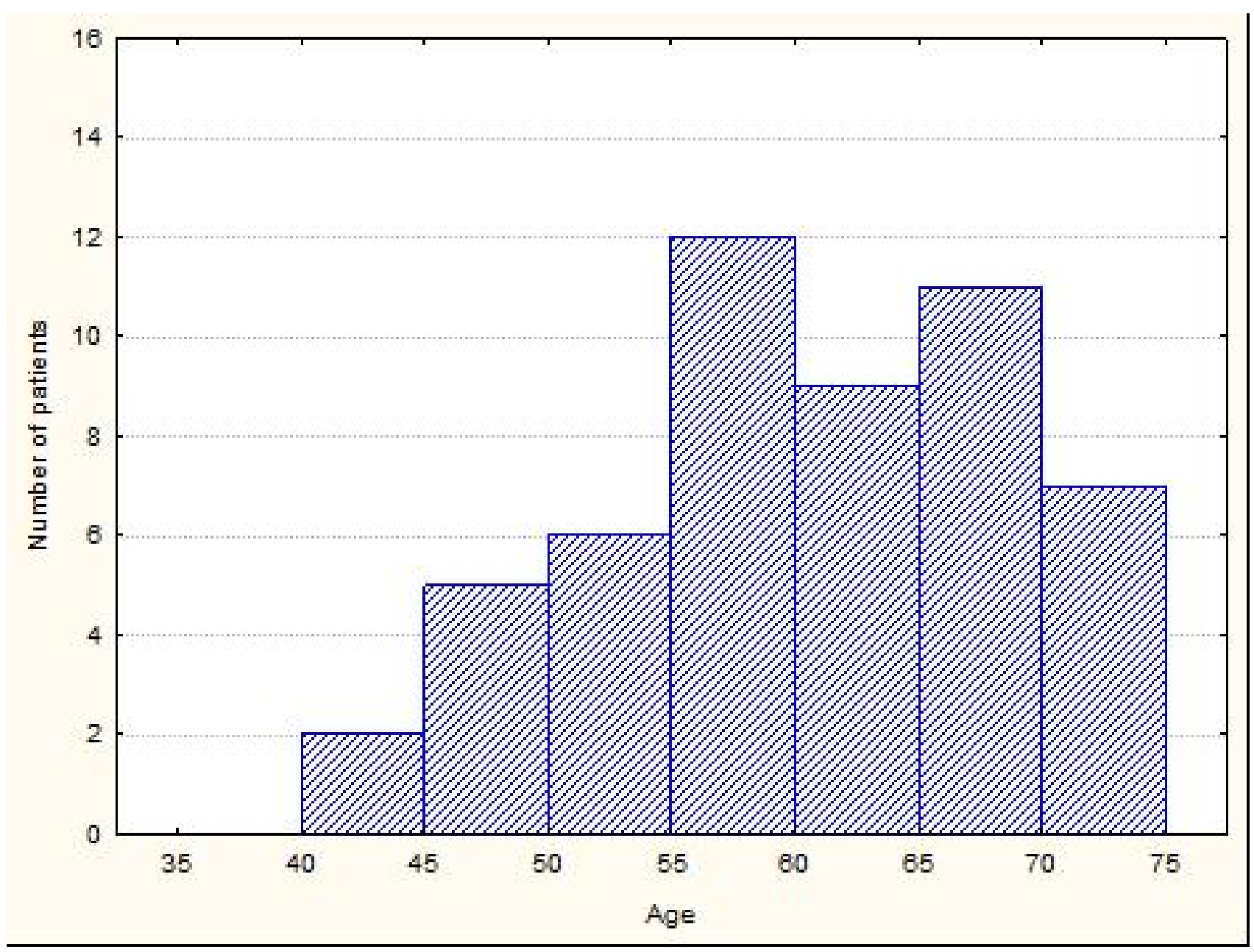

F I G U R E 1. Distribution of patients by age (in years): $x$ axis: age in years, $y$ axis: number of patients. (number of patients: 52, mean of age: 60,7, median of age 61,5, minimum 43 years, maximum 75 years, std.dev. 8.08).

A

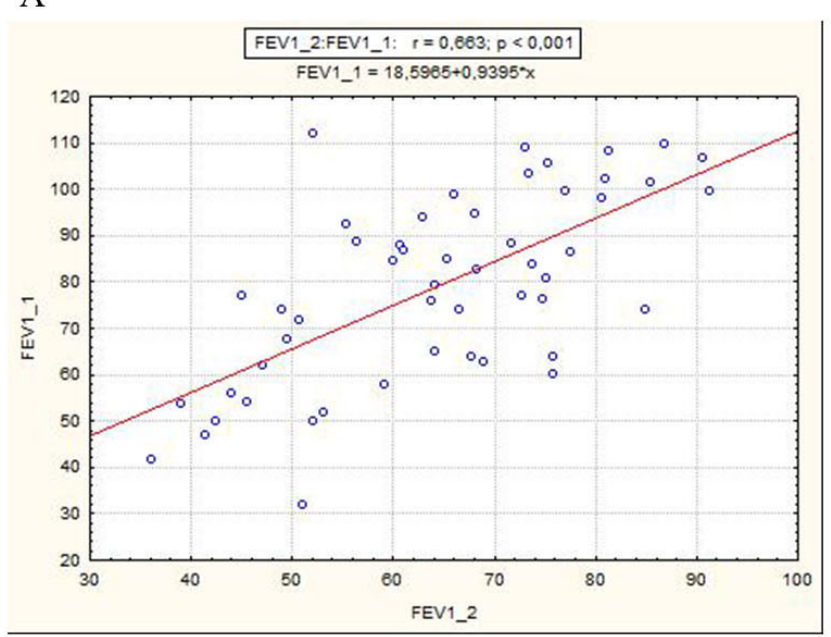

B

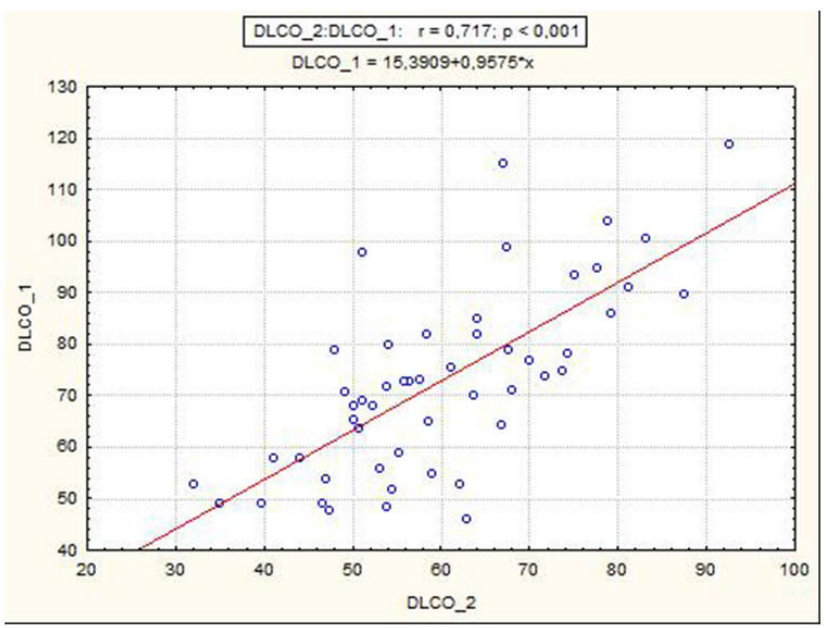

F I G U R E 2. Univariate analysis scatterplot showing the relationship between.

A) preoperative and postoperative FEV1 (FEV1_1 = preoperative FEV1, FEV1_2 = postoperative FEV1), $r=0.663, p<$ 0.001 . B) preoperative and postoperative $D L C O\left(D L C O \_1=\right.$ preoperative $D L C O, D L C O \_2=$ postoperative $\left.D L C O\right) . r=$ $0.717, p<0.001$.

by fissures [12]. There are many developmental variants in number and completeness of fissures and lobes [12].
Every lobe is divided in bronchopulmonal segments - real anatomical units of lungs with its own artery and bronchus 
A

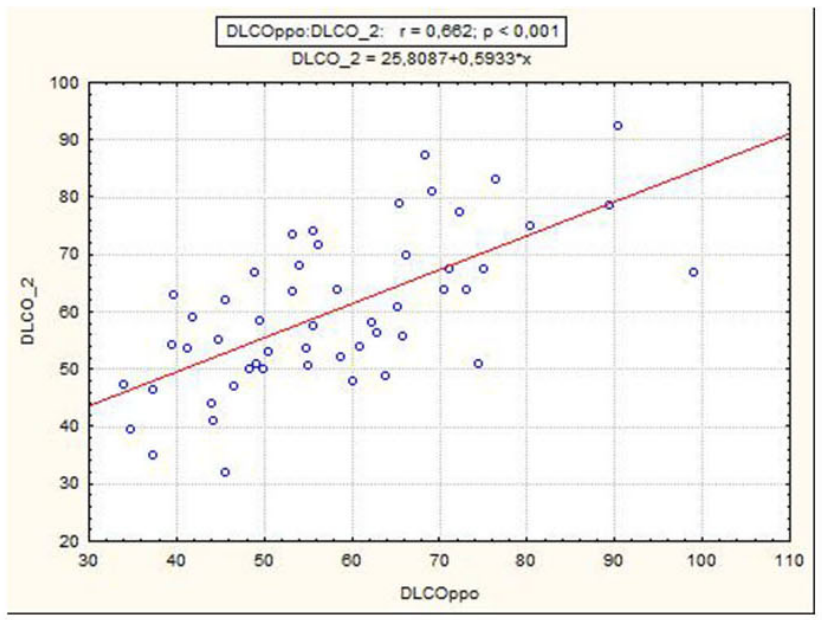

$\mathrm{B}$

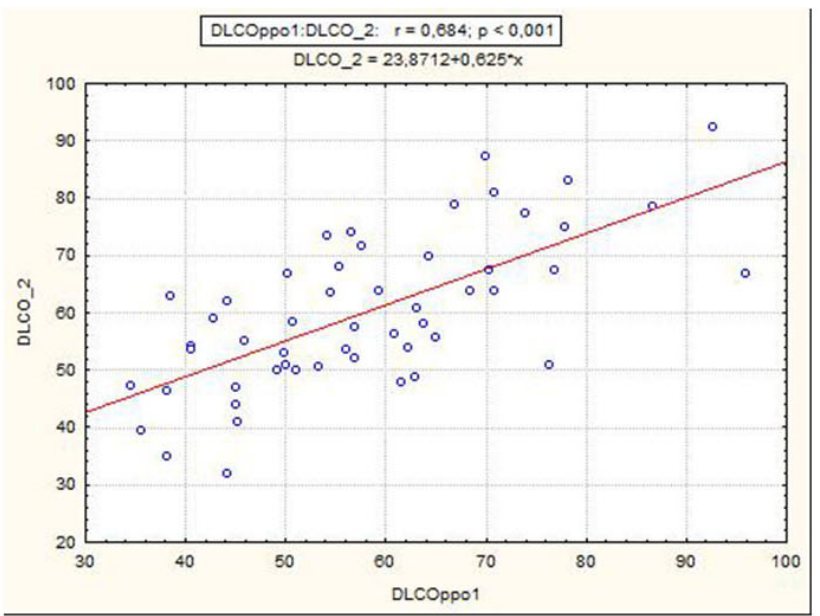

$\mathrm{C}$

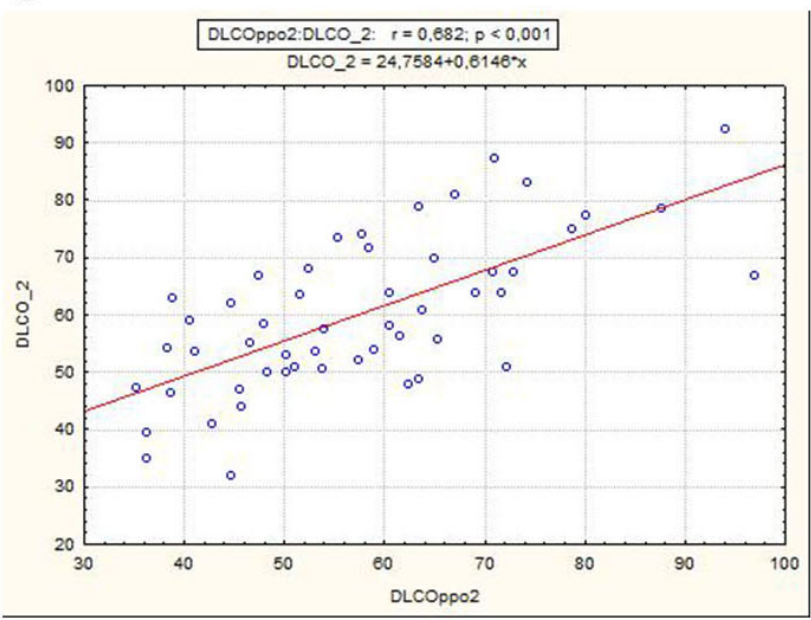

F I G U R E 3. Univariate regression scatterplots showing the values of:

A) postoperative DLCO and PpoDLCO - subsegment calculation (DLCO_2 = postoperative DLCO, DLCOppo = PpoDLCO - subsegments). $r=0.662, p<0.001$.

B) postoperative DLCO and PpoDLCO - 18 segments calculation (DLCO_2 = postoperative DLCO, DLCOppo1 = PpoDLCO - 18 segments). $r=0.684, p<0.001$.

C) postoperative DLCO and PpoDLCO - 19 segments calculation (DLCO_2 = postoperative DLCO, DLCOppo $2=$ PpoDLCO -19 segments). $r=0.682, p<0.001$.

[12]. Generally speaking, lungs have 18 segments: 10 segments right and 8 segments left with many variations [12]. Segments are divided in subsegments, altogether 42 subsegments in both lungs [10]. Boyden classification of segments shows that every lung, in fact, has 10 segments [13]. But, on left side first two segments share one bronchus (so called apicoposterior segment), and common bronchus exist for anterior and mediobasal segment - so we have anteromedial segment of left lung [13]. These fused segments appear as one [13].

According to it, there are some differences in subsegment and segment counting with respect of calculation of postoperative values. Subsegments are usually counted as follows: total subsegments: 42 , right upper lobe 6 , right middle lobe 4, right lower lobe 12, left upper lobe 10 and left lower lobe $10[1,10]$. Sawabata et al. mention counting of total lung segments as 18 (right upper lobe; 3 , right middle lobe; 2 , right lower lobe: 5 , left upper lobe 4 and left lower lobe 4) [4]. The others, as do Brunelli et al. in contemporary clinical guidelines, mostly counted total lung segments as 19 and count left upper lobe as 5 segments $[3,11]$. For lobectomy, following formula is suggested: PpoFEVI = preoperative FEVI $x(1-y / z)$, where y stands for the number of functional or unobstructed lung segments to be removed and $\mathrm{z}$ stands for total number of functional or unobstructed segments [2]. As common formula for calculation of PpoFEV1, PpoDLCO and even PpoVO2 (predicted postoperative oxygen uptake) we can find that Ppo values $=($ pre-operative value/T) $x R$ where $\mathrm{T}$ stands for total number of segments minus number of obstructed segments (estimated by image techniques and/or bronchoscopy) and R stands for $\mathrm{T}$ - number of functioning 
A

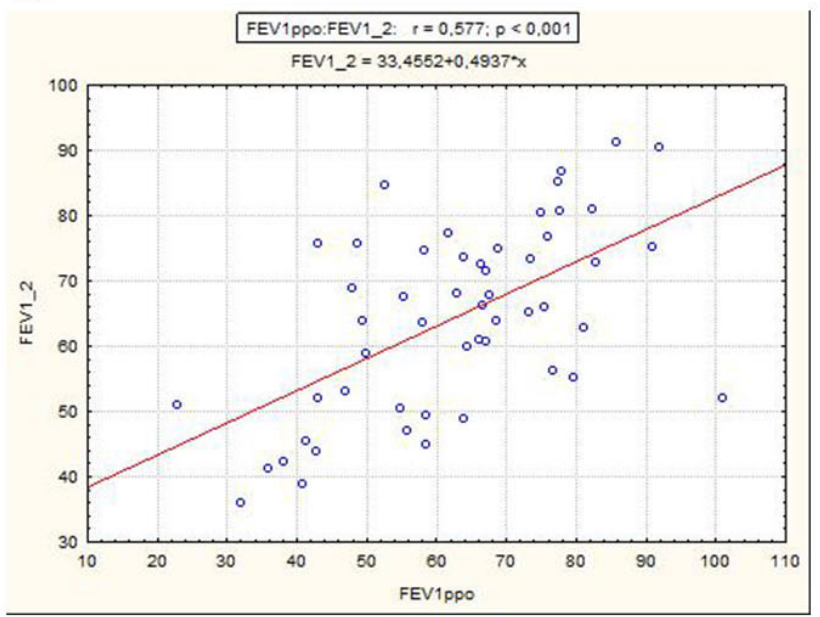

B

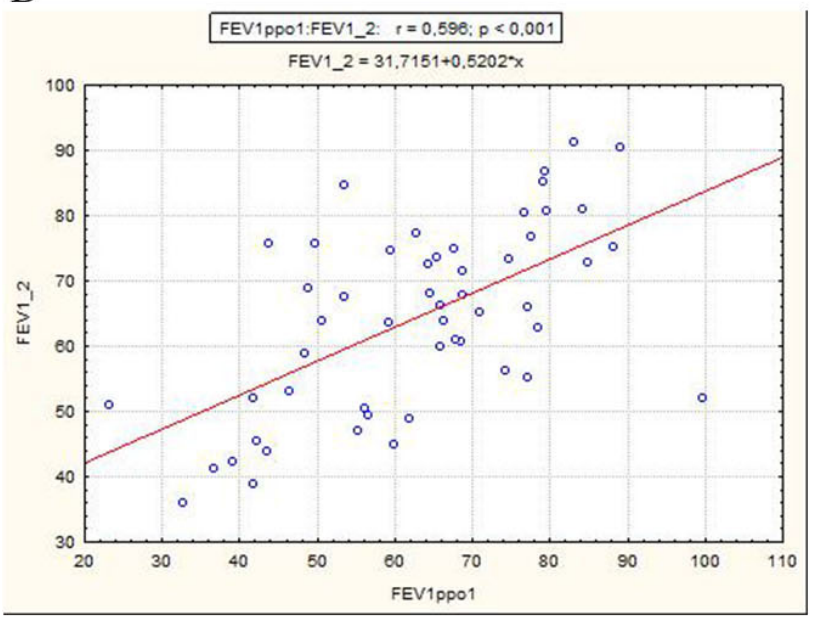

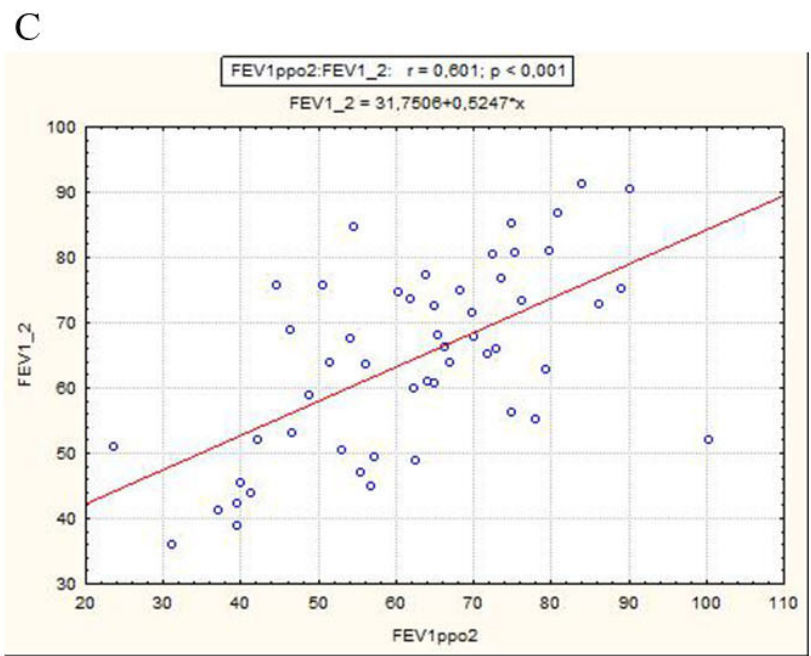

F I G U R E 4. Univariate regression scatterplots showing the values of:

A) postoperative FEV1 and PpoFEV1 - subsegments calculation (FEV1_2 = postoperative FEV1, FEV1ppo = PpoFEV1

$=$ subsegments). $r=0.577, p<0.001$.

B) postoperative FEVI and PpoFEV1 - 18 segments calculation (FEV1_2 = postoperative FEV1, FEV1ppo1 = PpoFEV1

-18 segments). $r=0.596, p<0.001$.

C) postoperative FEV1 and PpoFEV1 - 19 segments calculation (FEV1_2 = postoperative FEV1, FEV1ppo2 = PpoFEV1

-19 segments). $r=0.601, p<0.001$.

segments to be resected [11]. Nakahara et al. in the same manner suggest the following formula: PpoFEV1 = $(1-b / 42) x$ preop FEVI where $\mathrm{b}$ stands for number of subsegments in the resected lobe and 42 is the number of total subsegments [10]. Predicted postoperative values, according to guidelines, make basement for further testing $[2-4,11]$. The threshold is $40 \%$ or, more recently, $30 \%$ $[2-4,10,11]$. If PpoFEV1 and/or PpoDLCO are less than threshold, further and more complicated testing is recommended as a part of preoperative physiologic assessment for definitive identification of patients with increased risk of lung resection $[2-4,11]$.

\subsection{Hypothesis}

Segmental calculation method based on 19 segments is better than subsegmental method and segmental calculation method based on 18 segments in prediction of postoperative values of both FEV1 and DLCO one month after lung lobectomy.

\subsection{The aim of the study}

The aim of the study is to find out the best way of predicting of postoperative values of FEV1 and DLCO in means of simple calculations and, according to it, to help in risk assessment for patients undergone lung resection for lung carcinoma.

\section{Material and methods}

The study was approved by the Ethic Committee of Zagreb University Hospital Center. All patients signed informed consent to participate. The study is prospective. Data are 
A

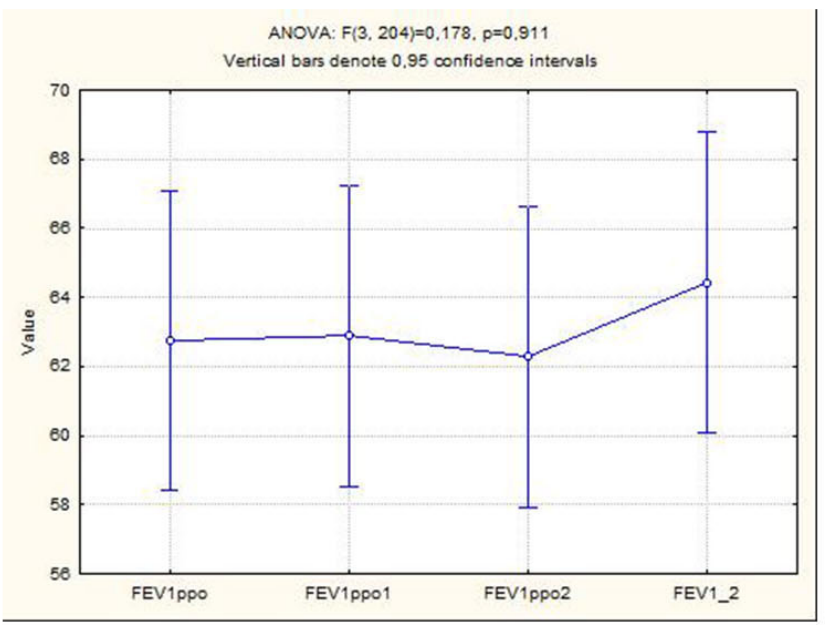

B

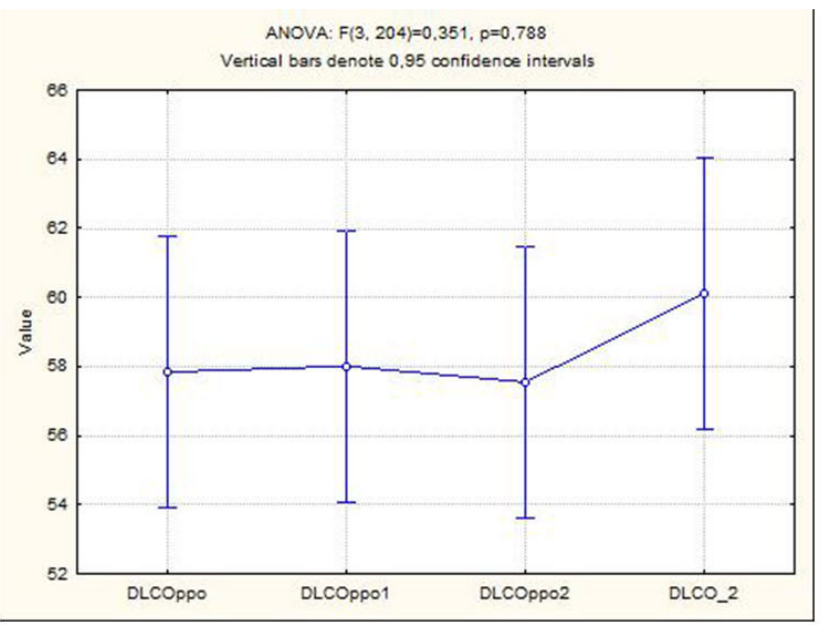

F I G U R E 5. Analysis of Variance (ANOVA):

A) $x$ axis: different FEV1 (FEV1ppo $=$ PpoFEV1 - subsegments, FEV1ppo1 $=$ PpoFEV1 -18 segments, FEV1ppo $2=$ PpoFEV1 - 19 segments, FEV1_2 = postoperative FEV1), y axis: values of FEV1 in percent predicted. Vertical bars denote 0.95 confidence intervals. $F=0.18, p=0.911(p>0.050)$.

B) $x$ axis shows DLCO (DLCOppo $=$ PpoDLCO - subsegments, DLCOppo1 $=P p o D L C O-18$ segments, DLCOppo $2=$ PpoDLCO - 19 segments, DLCO_2 = postoperative DLCO), $y$ axis shows values of DLCO in percent predicted. Vertical bars denote 0.95 confidence intervals. $F=0.35, p=0.788(p>0.050)$.

TA B L E 1. Distribution of patients by type of operation (absolute number and percent).

\begin{tabular}{|lcc|}
\hline Type of operation & Count & Percent \\
\hline RUL & 15 & 28.8 \\
\hline LLL & 8 & 15.4 \\
\hline LUL & 18 & 34.6 \\
\hline RLL & 7 & 13.5 \\
\hline RRML & 4 & 7.7 \\
\hline Total & 52 & 100.0 \\
\hline
\end{tabular}

$R U L=$ right upper lobectomy, $L L L=$ left lower lobectomy, $L U L=$ left upper lobectomy, $R L L=$ right lower lobectomy, $R R M L=$ resection of right medial lobe.

collected in from medical records of 52 patients that had undergone lung resection - lobectomy; no more, no less. Data included age, gender, FEV1 and DLCO values before surgery and during the first control survey one month after surgery. FEV1 and DLCO tests are part of regular preoperative risk assessment for any patient planned for lung resection in Jordanovac Department of Thoracic Surgery, Zagreb University Hospital Center. FEV1 and DLCO are routine standardized tests made by Laboratory for spirometry of Jordanovac Department for Lung Diseases, Zagreb University Hospital Center. PpoFEV1 and PpoDLCO are calculated according to simple formulas from literature, using segmental and subsegmental calculations $[1,4,10$, 11]. In this study we calculated postoperative values in three ways: as segmental with 18 segments, as segmental with 19 segments and with subsegmental method. Ob- structed segments/subsegments are estimated by means of bronchoscopy and/or CT scan and/or scintigraphy. The following equation is used: Ppo values $=$ (pre-operative value/T) $/ R$ where $\mathrm{T}$ is number of unobstructed subsegments or segments and $\mathrm{R}$ is the difference between $\mathrm{T}$ and number of unobstructed segments to be resected [11]. Calculated values are related to real postoperative values collected one month after surgery and statistically analyzed. Lung resections were performed through lateral thoracotomy. Preoperative survey, anesthesia (including thoracic epidural when feasible), postoperative analgesia, ICU admission, physiotherapy, mobilization, antibiotic and antithrombotic prophylaxis, treatment decisions and hospitalization were same as for patients not included in study.

\subsection{Statistical analysis}

Statistical analysis was performed using the STATISTICA software package version 6.1, SN AGA304B211928E61, StatSoft Inc.; USA. A sample of patients was analyzed by descriptive statistics and frequency tables. The observed parameters were analyzed by descriptive statistics. For the relationship between preoperative FEV1, calculated FEV1 and postoperative FEV1 and for the relationship between preoperative DLCO, calculated DCLO and postoperative DCLO, univariate regression analysis was used. Analysis of Variance (ANOVA) was used to test statistical difference between calculated PpoFEV1 and PpoDLCO and real postoperative values of FEV1 and DLCO. The results obtained by statistical analysis are shown graphically and numerically (tabular). Statistical testing is performed at a significance level of $95 \%(\alpha=0.05)$. 
TA B L E 2. Descriptive statistics of preoperative, calculated and postoperative FEV1 and DLCO: observed parameters are normally distributed (Skewness and Kurtosis are between $\mathbf{- 1}$ and 1 ).

\begin{tabular}{|c|c|c|c|c|c|c|c|c|}
\hline \multicolumn{9}{|c|}{ Descriptive Statistics } \\
\hline & $\mathbf{N}$ & Mean & Median & Minimum & Maximum & Std.Dev. & Skewness & Kurtosis \\
\hline FEV1_1 & 52 & 79.1 & 80.3 & 32 & 112.1 & 20.19 & -0.25 & -0.8 \\
\hline DLCO_1 & 52 & 73 & 72.5 & 46.1 & 119 & 17.74 & 0.54 & -0.08 \\
\hline FEV1ppo & 52 & 62.8 & 64.1 & 22.7 & 100.9 & 16.66 & -0.12 & -0.3 \\
\hline DLCOppo & 52 & 57.8 & 55.6 & 33.9 & 99 & 14.81 & 0.62 & 0.15 \\
\hline FEV1ppo1 & 52 & 62.9 & 64.9 & 23.1 & 99.6 & 16.32 & -0.18 & -0.38 \\
\hline DLCOppo1 & 52 & 58 & 56.7 & 34.5 & 95.9 & 14.53 & 0.56 & -0.03 \\
\hline FEV1ppo2 & 52 & 62.3 & 64 & 23.6 & 100.3 & 16.33 & -0.12 & -0.29 \\
\hline DLCOppo2 & 52 & 57.6 & 56.4 & 35.2 & 96.9 & 14.73 & 0.65 & 0.17 \\
\hline FEV1_2 & 52 & 64.4 & 65.6 & 36 & 91.3 & 14.24 & -0.12 & -0.85 \\
\hline DLCO_2 & 52 & 60.1 & 58.5 & 32 & 92.5 & 13.28 & 0.29 & -0.2 \\
\hline
\end{tabular}

1FEV1_1 = preoperative FEV1, DLCO_1 = preoperative DLCO, FEV1ppo = calculated postoperative FEV1 by subsegmental method, DLCOppo = calculated postoperative DLCO by subsegmental method, FEV1ppo1 = calculated postoperative FEV1 by segmental method (18 segments), DLCOppo1 = calculated postoperative DLCO by segmental method (18 segments), FEV1ppo2 = calculated postoperative FEV1 by segmental method (19 segments), DLCOppo2 = calculated postoperative DLCO by segmental method (19 segments), FEV1_2 = real postoperative FEV1, DLCO_2 = real postoperative DLCO.

TA B L E 3. Analysis of Variance (ANOVA).

\begin{tabular}{|lcccc|} 
Parameter & N & Mean & Std.Dev. & Std.Err. \\
\hline FEV1ppo & 52 & 62.7 & 16.66 & 2.31 \\
\hline FEV1ppo1 & 52 & 62.9 & 16.32 & 2.26 \\
\hline FEV1ppo2 & 52 & 62.3 & 16.33 & 2.26 \\
\hline FEV1_2 & 52 & 64.4 & 14.24 & 1.98 \\
\hline DLCOppo & 52 & 57.8 & 14.81 & 2.05 \\
\hline DLCOppo1 & 52 & 58 & 14.53 & 2.02 \\
\hline DLCOppo2 & 52 & 57.5 & 14.73 & 2.04 \\
\hline DLCO_2 & 52 & 60.1 & 13.28 & 1.84 \\
\hline
\end{tabular}

FEV1 (FEV1ppo = PpoFEV1 - subsegments, FEV1ppo1 = PpoFEV1 - 18 segments, FEV1ppo 2 = PpoFEV1 19 segments, FEV1_2 = postoperative FEV1): mean, standard deviation and standard error. DLCO (DLCOppo = PpoDLCO - subsegments, DLCOppo1 = PpoDLCO18 segments, DLCOppo2 = PpoDLCO - 19 segments, DLCO_2 = postoperative DLCO); mean, standard deviation and standard error.

\section{Results}

Total of 52 patients were $67.3 \%$ male (or 35 patients) and $32.7 \%$ female (or 17 patients).

Distribution of patients by age and type of surgery is shown in Table 1 and Fig. 1. Based on the results of the descriptive statistics of preoperative, calculated and postoperative FEV1 and DLCO, it can be concluded that the values of observed parameters are normally distributed (Skewness and Kurtosis are between -1 and 1), so that parametric statistical methods can be applied (Table 2).

Univariate regression analysis shows statistically significant correlation $(\mathrm{p}<0.001)$ between all dependent variables and their independent variables (Fig. 2, 3, 4). As expected, correlation coefficient, $r$, as a measure of strength of relationship, show the highest values (closest to 1) in correlation analysis between preoperative FEV1/DLCO values and calculated PpoFEV1/PpoDLCO values $(r=0.954-$ 0.975 , not shown in figures).

Correlation coefficient $r$ is not so high (0.577-0.717) in univariate regression analysis of relationship between PpoFEV1/PpoDLCO values and observed real postoperative FEV1/DLCO values (Fig. 3, 4).

Preoperative and postoperative DLCO correlate most significantly with PpoDLCO - 18 segments method, then with PpoDLCO - 19 segments method, and PpoDLCO subsegmental method (Fig. 3). Preoperative FEV correlates most significantly with PpoFEV1 - 18 segments method, then PpoFEV1 - 19 segments method, and PpoFEV1 subsegmental method.

Postoperative measured FEV1 most significantly correlates with PpoFEV1 - 19 segments method, then PpoFEV1 - 18 segments method, and PpoFEV1 subsegmental method (Fig. 4).

Relationships between preoperative and postoperative DLCO and FEV1 are shown in Fig. 2. Relationship between PpoDLCO (18 segments) and postoperative DLCO, and between PpoFEV1 - segmental method (19 segments) and postoperative FEV1 are shown in Figs. 3 and 4. According to the univariate regression analysis, the calculations of PpoDLCO - 18 segments method and PpoFEV1 19 segments method most correspond to the postoperative 
truth (Figs. 3, 4).

Hypothesis that segmental calculation method based on 19 segments is better than subsegmental method and segmental calculation method based on 18 segments in prediction of postoperative values of both FEV1 and DLCO one month after lung lobectomy is partially confirmed; e.g. calculation method based on 19 segments is better than subsegmental method and segmental calculation based on 18 segments in prediction of postoperative values of FEV1 one month after lung lobectomy, but this is not true for DLCO.

Clinical significance of this finding is less clear because of small differences in observed values.

Analysis of Variance (ANOVA) shows that simple calculated PpoFEV1 and PpoDLCO underscore the real postoperative values of FEV1 and DLCO one month after lung resection - lobectomy, but this difference is not statistically significant ( $\mathrm{p}>0.050)$ (Table 3, Fig. 5)

\section{Discussion}

According to our study, real values of postoperative DLCO correlate most significantly with PpoDLCO calculated by segmental method (18 segments), but real values of postoperative FEV1 correlate most significantly with ppoFEV1 calculated by 19 overall segments segmental method, according to univariate analysis. Hypothesis that segmental method based on 19 segments provides the best way of calculation of Ppo values for both FEV1 and DLCO is not confirmed. In fact, the problem is in PpoDLCO calculation which seems to be better by 18 -segments calculation. As we can find in literature, it could be appropriate to take number of segments as 18 or 20 segments too [5, 12, 13]. Nakahara et al. count subsegments and it means 42 units as a basis of calculation [10]. Anyway, because of some special characteristics of lingula, left upper lobe is in contemporary guidelines taken as bigger then left lower lobe, and even of the same size as right lower lobe $[2,5,11]$. So Brunelli et al. in the most recent European guidelines, counted total lung segments as 19 with left upper lobe estimation as 5 segments [11]. Sawabata et al. in their Japanese guidelines count the lung segments in the same manner [5]. Most of preoperative calculations could be made by simple counting of nonobstructed segments or subsegments $[5,11]$. For lobectomy it even seems that we cannot get more by sophisticated methods like scintigraphy - different is for pre-pulmectomy calculations [5, 11]. Values of PpoFEV1 and PpoDLCO help us make a decision of proceeding with surgery or for need for further testing and postponing the surgery sometimes even more than a week. For patients with PpoFEV1 and/or PpoDLCO less than 70\% it is advised to go to further testing - those with PpoFEV1 and/or PpoDLCO less than $30 \%$ and associated insufficient exercise tolerance were deemed inoperable or undergone lesser resections where possible [7]. Cancer surgery is never really elective and we risk the worsening of the illness by postponing the surgery too far [1]. So it could be important to make risk assessment procedure as appropriate as possible. In this study we calculated postoperative values in three ways: as segmental with 18 segments, segmental with 19 segments and with subsegmental method, and, at least for FEV1, it seems that 19-segment counting works the best. Clinical significance of these results is possibly not great because of low differences between values got by different Ppo calculations.

We chose to measure postoperative values 1 month after surgery because, according to Brunelli et al., the prediction of FEV1 and DLCO after lobectomy using Ppo calculations according the British Thoracic Society recommendation are "almost perfect" after 1 month [4, 7]. British Thoracic Society recommended number of segments for calculation was 19 [4]. For comparison, Brunelli et al. in one other study found one month after lobectomy that mean PpoFEV1 was $72 \%$ and measured mean FEV1 71.4\%. For DLCO it was mean Ppo 63.5\% and real 64.5\% [8]. In our study, these mean values are: PpoFEV1 62.29\% vs. $64.435 \%$ and PpoDLCO $57.546 \%$ vs. $60.127 \%$ calculated by same method (Table 3.) Of course, there is much in favor of Brunelli et al.; larger number of patients for example [7, 8]. Anyway, according to our results, PpoFEV1 and PpoDLCO maybe underestimate the real postoperative values of FEV1 and DLCO one month after lung resection - lobectomy, but this difference is not found as statistically significant (Table 3. and Fig. 5.). Our analysis shows that postoperative FEV1 and DLCO will have values of $30 \%$ if preoperative FEV1 and DLCO are between $45 \%$ and $50 \%(\mathrm{p}<0.001$, Fig. 5.). According to recent guidelines, a value of $30 \%$ should be used to distinguish between normal risk and higher risk lung resection candidates [11]. However, values of PpoFEV1 and PpoDLCO should not be used alone for select patients for lung resection [11]. Exercise tolerance is regarded important for risk stratification and should be indicated in all patients with lower FEV1 and DLCO [11]. Values of FEV1 and DLCO change during the postoperative course and they are usually greater then calculated after a few months [7].

\section{Conclusions}

1. PpoFEV1 and PpoDLCO calculations are a part of risk assessment for patients undergoing lung resection for lung cancer.

2. Segmental and subsegmental methods of PpoFEV1 and PpoDLCO calculations shows statistically significant correlations with observed postoperative FEV1 and DLCO one month after lung resection for lung cancer. Differences among methods are of questioned clinical significance.

3. Among three methods of calculation (18-segments segmental, 19-segments segmental and subsegmental), calculation of PpoFEV1 by 19-segments segmental method is statistically closest to observed postoperative FEV1 one month after lung lobectomy for lung cancer.

4. Among three methods of calculation (18-segments segmental, 19-segments segmental and subsegmental), calculation of PpoDLCO by 18-segments segmental method 
is statistically closest to observed postoperative DLCO one month after lung lobectomy for lung cancer.

5. PpoFEV1 and PpoDLCO underscore observed postoperative FEV1 and DLCO one month after lung resection - lobectomy, but it is not statistically significant.

\section{Limitations}

1. It is a small study. Bigger study is needed to make any relevant conclusions.

2. There are differences in size of lobes removed that can be relevant; for example, right middle lobe contains less relevant pulmonary tissue than all other lobes. Our study includes patients with different resection sizes.

3. It could be important to address the calculations for pulmectomy. This study does not address this important question in thoracic surgery at all.

4. It is not the same if patients have chronic obstructive lung disease or not. Our study does not make that difference.

\section{ACKNOWLEDGMENTS}

We would like to express our gratitude to all colleagues who helped us during the writing of this manuscript, all the peer reviewers and editors for their opinions and suggestions, and, especially, to Miljenko Košiček for his help in statistical analysis.

\section{CONFLICT OF INTEREST}

The authors declare no conflict of interest.

\section{REFERENCES}

[1] Slinger P, Darling G. Preanesthetic assessment for thoracic surgery. In: Slinger P, editor. Principles and practice of anesthesia for thoracic surgery. New York etc. Springer; 2011.p.11-34.

[2] Brunelli A, Kim AW, Berger KI, Addrizzo-Harris DJ. Physiologic evaluation of the patient with lung cancer being considered for resectional surgery: diagnosis and management of lung cancer, 3rd ed: American College of Chest Physicians Evidence-Based Clinical Practice Guidelines. Chest. 2013;143:166-90.

[3] Choi H, Mazzone P. Preoperative evaluation of the patient with lung cancer being considered for lung resection. Curr Opin Anaesthesiol. 2015;28:18-25.

[4] British Thoracic Society and the Society of Cardiothoracic Surgeons of Great Britain and Ireland Working Party. BTS guidelines: guidelines on the selection of patients with lung cancer for surgery. Thorax. 2001;56:89-108.

[5] Sawabata N, Nagayasu T, Kadota Y, Goto T, Horio H, Mori T, et al. Risk assessment of lung resection for lung cancer according to pulmonary function: republication of systematic review and proposals by guideline committee of the Japanese Association for Chest Surgery 2014. Gen Thorac Cardiovasc Surg. 2015;63:14-211.

[6] Beckles MA, Spiro SG, Colice GL, Rudd RM. The physiologic evaluation of patients with lung cancer being considered for resectional surgery. Chest. 2003;123:105-14.

[7] Brunelli A, Refai M, Salati M, Xiume F, Sabbatini A. Predicted versus observed FEV1 and DLCO after major lung resection: a prospective evaluation at different postoperative periods. Ann Thorac Surg. 2007;83:1134-9.

[8] Brunelli A, Xiume F, Refai M, Salati M, Marasco R, Sciarra V, et al. Evaluation of expiratory volume, diffusion capacity, and exercise tolerance following major lung resection. Chest. 2007;131:141-7.

[9] Culver BH. Preoperative assessment of the thoracic surgery patient: pulmonary function testing. Semin Thorac Cardiovasc Surg. 2001;13:92.

[10] Nakahara K, Monden Y, Ohno K, Miyoshi S, Maeda H, Kawashima Y. A method for predicting postoperative lung function and its relation to postoperative complications in patients with lung cancer. Ann Thorac Surg. 1985;39:260-5.

[11] Brunelli A, Charloux A, Bolliger CT, Rocco G, Sculier JP, Varela G, et al. ERS/ESTS clinical guidelines on fitness for radical therapy in lung cancer patients (surgery and chemo-radiotherapy). Eur Respir J. 2009;34:17-41

[12] Baue AE. Chest wall, pleura, lungs and diaphragm. In: Davis JH, editor: Clinical surgery. St. Louis etc: The C.V. Mosby Company; 1987. p.1190-272.

[13] Boyden EA. A critique of the international nomenclature on bronchopulmonary segments. Diseases of the chest. 1953;23:266-9.

How to cite this article: Vjekoslav Karadža, Ivanka Karadža. Comparison of Three Methods of Predictive Postoperative FEV1 and DLCO Calculations in Relation to Their Observed Postoperative Values in Lung Resection. Signa Vitae. 2020;16(1):25-32. doi:10.22514/sv.2020.16.0004. 